\title{
JWood: Um Objeto Educacional para Aprendizado de Elementos de Estruturas de Madeira
}

\section{JWood: Learning Object for Elements of Timber Structures}

\author{
Juliano Lima da Silva' ${ }^{1}$ Zacarias Martin Chamberlain Pravia ${ }^{2}$
}

\begin{abstract}
Resumo
Este trabalho apresenta o objeto educacional JWood, um aplicativo programado em Java que busca atualizar conceitos de engenharia na área de dimensionamento de elementos de estrutura de madeira, mais um programa do projeto educacional Etools (www.etools.upf.br). O aplicativo tem como foco principal o ensino de disciplinas relacionadas à Estruturas de Madeira, seguindo os métodos propostos na revisão com votação nacional aprovada da ABNT NBR 7190/2012 - Projetos de Estruturas de Madeira e buscando apresentar seus conceitos ao usuário de uma maneira dinâmica, organizada passo a passo com a lógica de procedimento manual, executando cálculos referentes à tração, compressão, flexão e flexocompressão de elementos de madeira, gerando inclusive ao final destes, um relatório detalhado que apresenta as operações realizadas.
\end{abstract}

Palavras-chave: Estruturas de Madeira. Objeto Educacional. Programação.

\begin{abstract}
This paper presents the educational object JWood, a Java programmed application that seeks to update engineering concepts in the computational design of timber elements, it is another software of the educational project Etools (www.etools.upf.br). The application has as main focus the learning of disciplines related to Timber Structures, following the proposed by methods in the revision of ABNT NBR 7190/2012 Wooden structures projects and looking to present its concepts to the user in a dynamic, organized and easily comprehensive way, executing calculations related to traction, compression, bending and bending combined with axial compression of timber elements, including the generation of a detailed report that presents the performed operations.
\end{abstract}

Keywords: Timber Structures. Educational Object. Programming.

\footnotetext{
${ }^{1}$ Me. Arquitetura e Urbanismo, Depto de Engenharia Civil, IMED, RS, Brasil; E-mail: juliano_lima_silva@ @otmail.com

${ }^{2}$ Dr. Prof., Depto de Engenharia Civil, PPGEng, UPF, RS, Brasil; E-mail: zacarias@upf.br
} 


\section{Introdução}

O ensino de estruturas no ramo de engenharia civil possui um alto nível de abstração, o que pode dificultar o aprendizado de alguns conceitos e fundamentos, além de exigir conhecimentos cuja compreensão é difícil para grande parte dos alunos. Portanto, uma das alternativas propostas para proporcionar maior alcance nestes quesitos é o uso do computador na educação de disciplinas de estruturas, através de objetos educacionais, segundo (KRIPKA; PRAVIA, 1999).

Por ser um material cujas características inerentes o tornam propício para o uso estrutural, a madeira possui grande potencial no ramo da engenharia civil, pois, além de renovável e de preço competitivo, é naturalmente adequada para resistir esforços de tração e compressão paralela às fibras, bem como um material de ótima relação peso-resistência, cujo uso torna-se continuamente mais presente na atualidade, onde cada vez mais é almejada a criação de soluções sustentáveis que combinem qualidade com segurança.

O aplicativo a ser apresentado chama-se JWood, cujo campo de aplicação destina-se ao ensino esquematizado de estruturas de madeira. Seu objetivo é proporcionar à estudantes de engenharia, professores e profissionais da área uma ferramenta que os auxilie na tomada de decisões, apresentando os conceitos inseridos na NBR 7190/2012 - Projetos de Estruturas de Madeira de uma forma simples e descomplicada. $\mathrm{O}$ aplicativo possui equações para situações de peças biapoiadas e com carregamentos distribuídos.

\section{Revisão da NBR 7190/2012}

A antiga NBR 7190/1997, passou por um projeto de revisão cuja aprovação previa a substituição desta de acordo com informações atualizadas. Sua renovação foi aprovada e diagramada conforme as regras da ABNT no ano de 2012, consolidando assim alterações, principalmente no modelo de dimensionamento à compressão.

Este aplicativo busca adequar o contexto da revisão da norma para o campo educacional, delimitando resumidamente as transparências de forma a facilitar sua interação com os usuários.

\section{O aplicativo JWOOD}

Nascido do Trabalho de Conclusão de Curso de um dos autores, o aplicativo JWood teve o início de seu desenvolvimento no fim de 2014.
Sua interface e código foram implementados na linguagem Java de programação, cujo design e programação foram feitos com auxílio da IDE Netbeans 8.0.2. O aplicativo conta com uma interface composta de abas separadas por etapas, onde o usuário inicialmente é apresentado a uma tela principal, figura 1 , que revela a versão atual instalada e traz informações ao usuário pelo botão "Sobre", figura 2 .

Figura 1: Tela Inicial

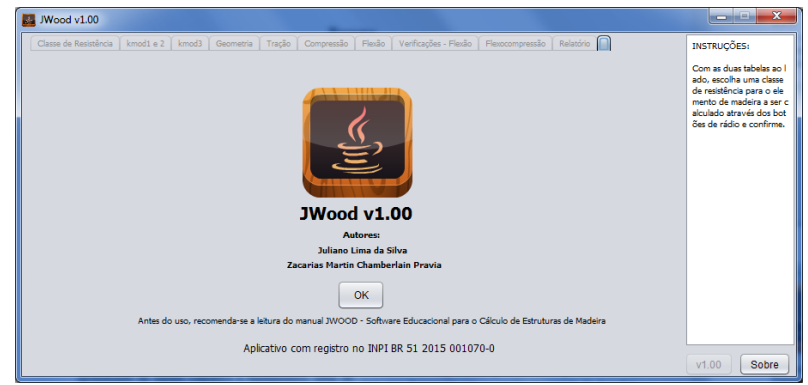

Fonte: Os autores

Figura 2: Menu Sobre

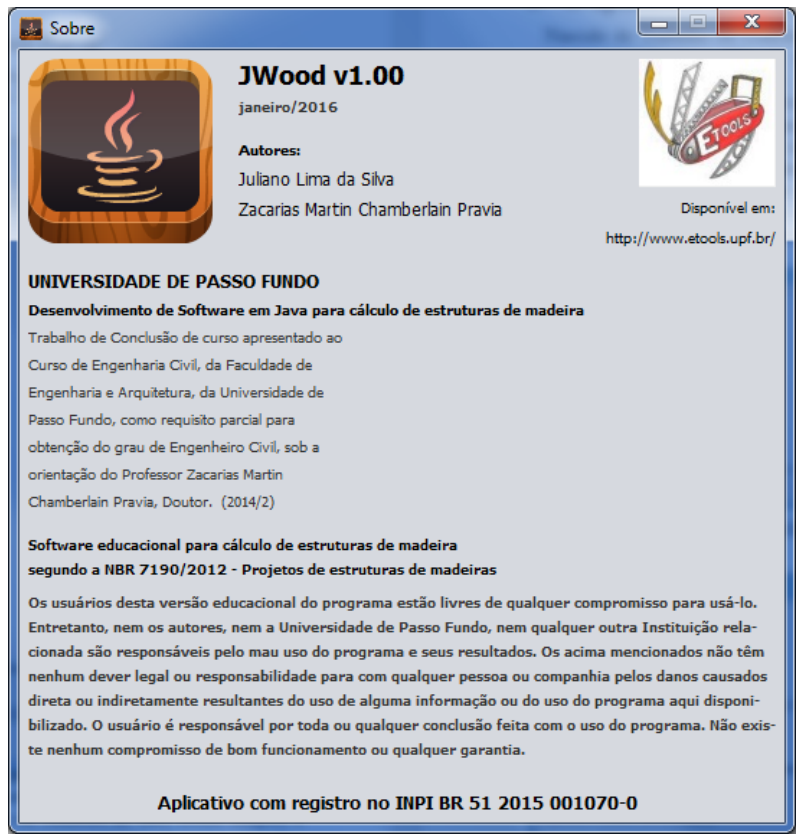

Fonte: Os autores

$\mathrm{O}$ aplicativo funciona de maneira simples, que, em resumo, consiste na aplicação de conceitos da NBR 7190/2012, apresentados de maneira sequencial, organizada e descomplicada, a fim de guiar o usuário através dos processos de escolha do material, definição de parâmetros e execução de cálculos relativos à resistência a esforços do elemento a ser dimensionado. 
Seu campo de aplicação abrange o dimensionamento de elementos de madeira, bem como a verificação destes quando submetidos a verificações de cálculo apresentadas pela NBR 7190/2012, possibilitando o dimensionamento de uma variedade de estruturas, elementos ou peças de madeira quando solicitados a esforços de tração e compressão, bem como análise e verificação de elementos submetidos à flexão e flexocompressão.

\section{Definição de propriedades}

É errônea a impressão de que a madeira estrutural é um material frágil e que queima com facilidade. Com as novas tecnologias de tratamento, o material torna-se não somente durável como extremamente resistente a efeitos externos.

Segundo a NBR 7190/2012, a padronização da madeira em classes estruturais auxilia na orientação da escolha do material adequado durante a elaboração de projetos e tomada de decisões. Tendo em vista a convergência de JWood com os aspectos apresentados na norma, as tabelas do item 4.3.5 da NBR foram incorporadas no aplicativo.

O usuário inicialmente deve definir qual classe de madeira irá utilizar no elemento a ser calculado, através das tabelas 1 e 2 :

Tabela 1: Classes de Resistência - Coníferas

\begin{tabular}{ccccc}
\hline \multicolumn{5}{c}{ Coníferas } \\
\multicolumn{6}{c}{ (Valores na condição-padrão de referência $U=12 \%)$} \\
\hline Classes & $f_{c 0, k}$ & $f_{v 0, k}$ & $E_{c 0, m}$ & $\rho_{\text {aparente }}$ \\
\hline C20 & 20 & 4 & 3500 & 500 \\
C25 & 25 & 5 & 8500 & 550 \\
C30 & 30 & 6 & 14500 & 600 \\
\hline
\end{tabular}

Fonte: Os autores.

Tabela 2: Classes de Resistência - Folhosas

Folhosas

(Valores na condição-padrão de referência $U=12 \%$ )

\begin{tabular}{ccccc}
\hline Classes & $f_{c 0, k}$ & $f_{v 0, k}$ & $E_{c 0, m}$ & $\rho_{\text {aparente }}$ \\
\hline D20 & 20 & 4 & 9500 & 650 \\
D30 & 30 & 5 & 14500 & 800 \\
D40 & 40 & 6 & 19500 & 950 \\
D50 & 50 & 7 & 22000 & 970 \\
D60 & 60 & 8 & 24500 & 1000 \\
\hline
\end{tabular}

Fonte: Os autores.
Portanto, na primeira aba de JWood, figura 3, o usuário seleciona a classe definida entre coníferas e folhosas, por meio de botões de rádio. Neste momento, o aplicativo estará gravando em seu interior as variáveis apresentadas nas tabelas 1 e 2, para uso posterior.

Figura 3: Aba de classes de resistência

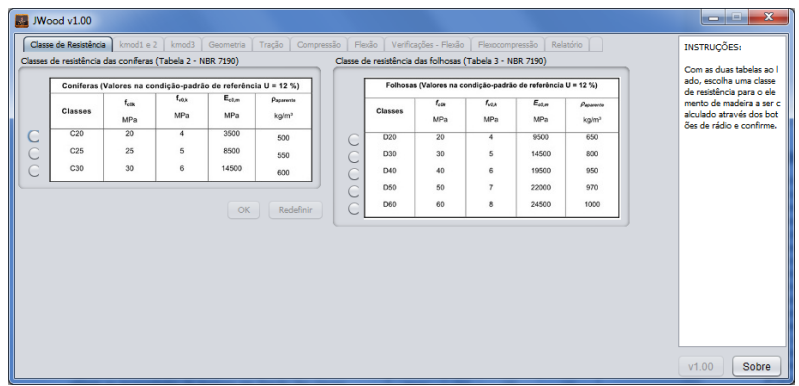

Fonte: Os autores.

O passo seguinte à definição da classe de resistência é referente à determinação dos coeficientes de modificação, expressos no item 4.4.4 da NBR 7190/2012, cujos valores afetam as propriedades da madeira em função das classes de carregamento, umidade e do emprego de madeira classificada ou de segunda qualidade.

O usuário então deve, guiado pelas tabelas da norma, tabelas 3 e 4, inserir nos campos em amarelo, figura 4, valores referentes aos dados que deseja assumir em seu cálculo, utilizando as tabelas como referência para definir os valores de $k \bmod 1$ e $\operatorname{kmod} 2$.

Figura 4: Aba de kmod1 e kmod2

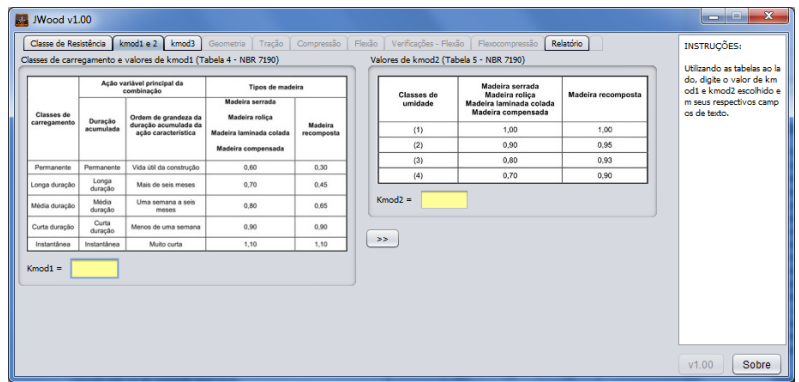

Fonte: Os autores.

Ao definir os coeficientes relativos à classe de carregamento e umidade, o usuário deve avançar à aba do coeficiente de classe de qualidade, na aba kmod3, figura 5. 
Tabela 3: Valores do coeficiente de carregamento

\begin{tabular}{ccccc}
\hline \multirow{2}{*}{$\begin{array}{c}\text { Classes de } \\
\text { carregamento }\end{array}$} & $\begin{array}{l}\text { Ação viável principal da combinação } \\
\text { acumulada }\end{array}$ & $\begin{array}{l}\text { Ordem de grandeza da dura- } \\
\text { ção acumulada da ação ca- } \\
\text { racterística }\end{array}$ & $\begin{array}{l}\text { Madeira serrada, Madeira roliça, } \\
\text { Madeira laminada colada, Ma- } \\
\text { deira compensada }\end{array}$ & $\begin{array}{l}\text { Madeira } \\
\text { recom- } \\
\text { posta }\end{array}$ \\
\hline Permanente & Permanente & Vida útil da Construção & 0.60 & 0.30 \\
Longa duração & Longa duração & Mais de 6 meses & 0.70 & 0.45 \\
Média duração & Média duração & Uma semana a seis meses & 0.80 & 0.65 \\
Curta duração & Curta duração & Menos de uma semana & 0.90 & 0.90 \\
Instantânea & Instantânea & Muito curta & 1.10 & 1.10 \\
\hline
\end{tabular}

Fonte: Os autores.

Tabela 4: Valores do coeficiente de umidade.

\begin{tabular}{ccc}
\hline $\begin{array}{c}\text { Classes de } \\
\text { umidade }\end{array}$ & $\begin{array}{c}\text { Madeira serrada } \\
\text { Madeira roliça } \\
\text { Madeira laminada colada } \\
\text { Madeira compensada }\end{array}$ & $\begin{array}{c}\text { Madeira } \\
\text { recomposta }\end{array}$ \\
\hline$(1)$ & 1.00 & 1.00 \\
$(2)$ & 0.90 & 0.95 \\
$(3)$ & 0.80 & 0.93 \\
$(4)$ & 0.70 & 0.90 \\
\hline
\end{tabular}

Fonte: Os autores.

Figura 5: Aba de kmod3

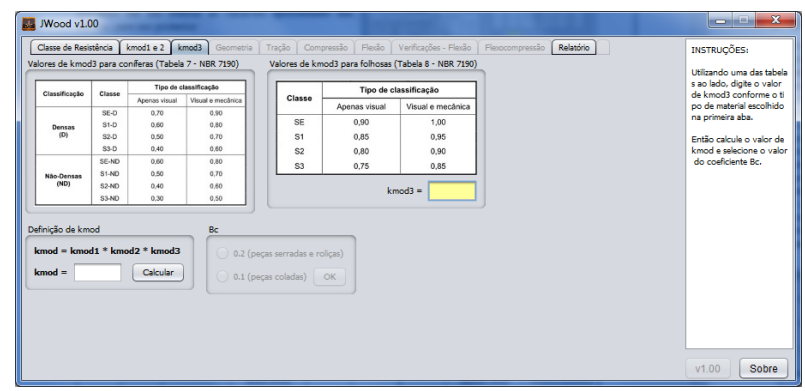

Fonte: Os autores.

Tabela 5: Valores do coeficiente de qualidade - Coníferas

\begin{tabular}{cccc}
\hline Classificação & Classe & \multicolumn{2}{c}{ Tipo de classificação } \\
\cline { 3 - 4 } & & $\begin{array}{l}\text { Apenas } \\
\text { visual }\end{array}$ & $\begin{array}{l}\text { Visual } \\
\text { e mecâ- } \\
\text { nica }\end{array}$ \\
\hline \multirow{2}{*}{ Densas } & SE-D & 0.70 & 0.90 \\
(D) & S1-D & 0.60 & 0.80 \\
& S2-D & 0.50 & 0.70 \\
& S3-D & 0.40 & 0.60 \\
Não-Densas & SE-ND & 1.60 & 1.80 \\
(ND) & S1-ND & 0.50 & 0.70 \\
& S2-ND & 0.40 & 0.60 \\
& S3-ND & 0.30 & 0.50 \\
\hline
\end{tabular}

Fonte: Os autores.
Tabela 6: Valores do coeficiente de qualidade - Folhosas

\begin{tabular}{ccc}
\hline Classes & \multicolumn{2}{c}{ Tipo de classificação } \\
\cline { 2 - 3 } & Apenas visual & Visual e mecânica \\
\hline SE & 0.90 & 1.00 \\
S1 & 0.85 & 0.95 \\
S2 & 0.80 & 0.90 \\
S3 & 0.75 & 0.85 \\
\hline
\end{tabular}

Fonte: Os autores

$\mathrm{Na}$ aba kmod3, figura 5, basta consultar novamente as tabelas 4 e 5, incorporadas da norma, digitando no campo em amarelo um valor relativo à qualidade e classificação (apenas visual ou visual e mecânica) da madeira a ser calculada.

Caso o usuário digite um número que não se encontra no intervalo de valores disponível em quaisquer das tabelas, ele será avisado e será solicitada a revisão dos valores digitados.

Depois de digitados os valores nos campos de texto amarelos, o usuário poderá calcular o coeficiente final de redução kmod, bem como definir o valor do fator para peças Bc, citado no item 5.5.4 da NBR 7190/2012.

\section{Dados Geométricos}

Após definidos todos os parâmetros de classe e coeficientes de redução do material a ser calculado, o programa irá orientar o usuário para as fases de dimensionamento e cálculo propriamente ditas.

O primeiro passo consta no pré-dimensionamento do elemento de madeira e a realização de alguns cálculos relacionados à geometria, que serão abordados posteriormente nas abas de cálculo.

Para definir a peça, basta o usuário digitar nos campos em amarelo, figura 6, valores pertinentes às dimensões base, altura e comprimento, solicitando então o cálculo da 
área.

Figura 6: Inserção de dados geométricos
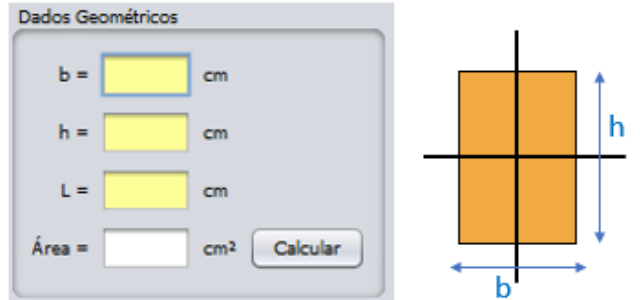

Fonte: Os autores.

É importante ressaltar que o valor mínimo a ser inserido é de $5 \mathrm{~cm}$ para as dimensões transversais, bem como uma área mínima resultante de $50 \mathrm{~cm}^{2}$. Caso o usuário insira valores inválidos ou menores que os condicionados, o programa irá alerta-lo.

Os procedimentos seguintes são liberados sequencialmente após a definição da área, para preparar o caminho passo-a-passo das equações pertinentes e guiar o usuário. Discriminado no item 5.5.2 da NBR 7190/2012, é necessário calcular a esbeltez do elemento, tanto no eixo $x$ quanto no eixo $y$, levando em consideração o comprimento de flambagem $L$ :

$$
\lambda_{x}=\frac{L}{\sqrt{\frac{h^{2}}{12}}} .
$$

A esbeltez em $x$ deve respeitar o limite máximo de 140 , desta forma, quesito que, caso não atendido, não permitirá que o usuário continue nos cálculos desta aba. $\mathrm{O}$ mesmo deve ser feito para a esbeltez em $y$ :

$$
\lambda_{y}=\frac{L}{\sqrt{\frac{b^{2}}{12}} .}
$$

O usuário será alertado, e poderá redefinir os valores caso a peça a ser dimensionada não passe na verificação de esbeltez. O próximo passo é a definição da inércia nos eixos, através das equações:

$$
\begin{aligned}
& I_{x}=\frac{b * h^{3}}{12}, \\
& I_{y}=\frac{h * b^{3}}{12} .
\end{aligned}
$$

Por fim, o módulo de resistência é calculado

$$
\begin{aligned}
& W_{x}=\frac{b * h^{2}}{6} \\
& W_{y}=\frac{h * b^{2}}{6}
\end{aligned}
$$

e as abas de cálculo, figura 7, são disponibilizadas.
Figura 7: Aba de Geometria

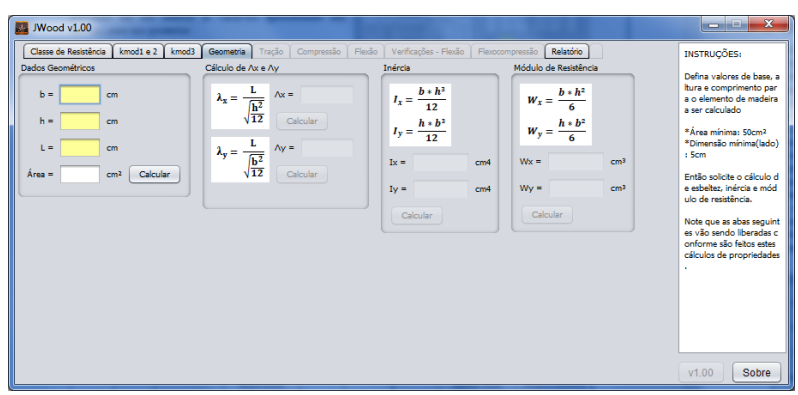

Fonte: Os autores.

\section{Tração paralela às fibras}

Segundo a NBR 7190/2012, o dimensionamento de um elemento de madeira passa pela equação geral de resistência característica, que aplica o coeficiente de modificação kmod, à resistência proveniente da classe do material escolhida e o coeficiente de minoração $\gamma_{w}$, de valor 1.8 para peças tracionadas e 1.4 para peças comprimidas ou flexionadas, como expresso no item 4.4.3 da norma NBR, através da fórmula:

$$
f_{c 0 d}=k_{\text {mod }} * \frac{f_{c 0 k}}{\gamma_{w}} .
$$

Como o cálculo da resistência à tração de um elemento de madeira depende apenas de sua área, basta o usuário solicitar os cálculos no aplicativo através da equação

$$
N t_{d}=f_{c 0 d} * A .
$$

É importante ressaltar que os cálculos de tração, compressão e flexão são independentes, podendo o usuário mudar de aba, figura 8 , a qualquer momento depois de ter definido as propriedades e a geometria.

Figura 8: Aba de Tração

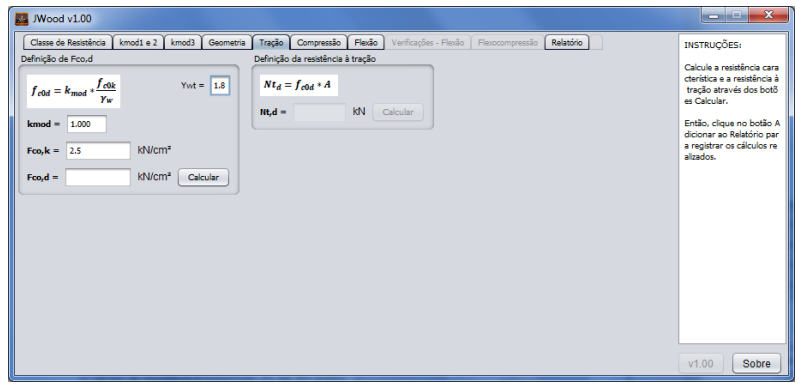

Fonte: Os autores.

\section{Compressão paralela às fibras}

O cálculo da resistência à compressão foi um dos pontos mais alterados na revisão da norma. A seguir é apresentada a sequência de cálculos pertinentes à sua obtenção. 
Depois de aplicada a equação geral de resistência, como expresso também em (5), basta o usuário solicitar os cálculos para que o programa o acompanhe a cada etapa, proporcionando facilidade de aprendizado.

O próximo passo é o cálculo do módulo de elasticidade característico, expresso no item 4.4.5 da NBR 7190/2012 através da fórmula:

$$
E_{0.05}=0.7 * E_{c 0 m}
$$

Com este módulo de elasticidade, o item 5.5.3 da NBR 7190/2012 prevê o cálculo da esbeltez relativa:

$$
\begin{aligned}
& \lambda_{\text {rel }, x}=\frac{\lambda_{x}}{\pi} * \sqrt{\frac{f_{c 0 k}}{E_{0.05}}}, \\
& \lambda_{\text {rel }, y}=\frac{\lambda_{y}}{\pi} * \sqrt{\frac{f_{c 0 k}}{E_{0.05}}} .
\end{aligned}
$$

Com o fator para peças Bc, definido na aba "kmod3", no início do programa, é possível calcular os coeficientes $k_{x}$ e $k_{y}$, como disposto no item 5.5.4 da NBR 7190/2012:

$$
\begin{aligned}
& k_{x}=0.5 *\left[1+\beta_{c} *\left(\lambda_{\text {relx }}-0.3\right)+\left(\lambda_{\text {rel }, x}\right)^{2}\right], \\
& k_{y}=0.5 *\left[1+\beta_{c} *\left(\lambda_{\text {rely }}-0.3\right)+\left(\lambda_{\text {rel }, y}\right)^{2}\right] .
\end{aligned}
$$

Em seguida, definem-se os coeficientes $k c_{x}$ e $k c_{y}$, relacionados à estabilidade das peças em solicitações de flexão ou compressão

$$
\begin{aligned}
& k c_{x}=\frac{1}{k_{x}+\sqrt{\left(k_{x}\right)^{2}-\left(\lambda_{\text {rel }, x}\right)^{2}}}, \\
& k c_{y}=\frac{1}{k_{y}+\sqrt{\left(k_{y}\right)^{2}-\left(\lambda_{\text {rel }, y}\right)^{2}}} .
\end{aligned}
$$

Finalmente, a resistência à compressão pode ser calculada a partir do menor valor encontrado em $x$ ou $y$, através de:

$$
N c_{d}=k c_{(x, y)} * f_{c 0 d} * A .
$$

Após executados todos os cálculos da aba de compressão, figura 9, o usuário poderá notar que foi liberada uma aba chamada "Flexocompressão", ferramenta à ser abordada no trabalho.

\section{Flexão e verificações}

O processo de cálculo à flexão é distinto dos de tração e flexão. Enquanto nestes é determinada a resistência ao esforço paralelo às fibras, o primeiro determinará se o elemento de madeira a ser calculado resiste aos carregamentos inseridos.
Figura 9: Aba de Compressão

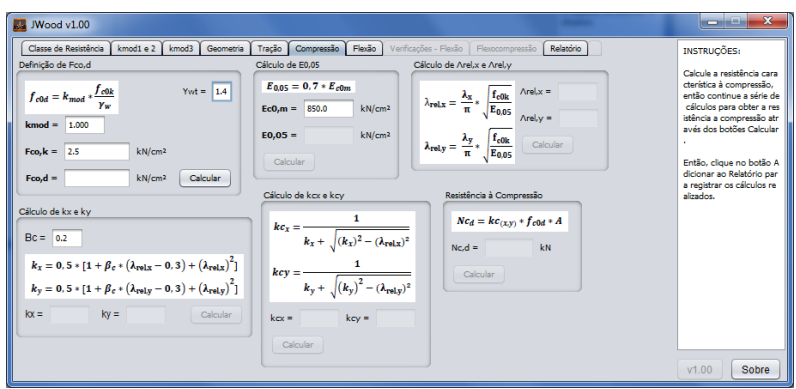

Fonte: Os autores

Primeiramente é necessário definir o módulo de elasticidade efetivo, disposto no item 4.4.9 da NBR 7190/2012, dado por:

$$
E_{c 0, e f}=k_{m o d} * E_{c 0 m}
$$

Então, o usuário deverá inserir nos campos em amarelo desta aba, figura 10, valores referentes aos carregamentos a serem aplicados na peça. Estes valores são de carga distribuída ao longo do comprimento da mesma, tendo natureza permanente, acidental e de vento. O usuário pode ainda inserir um valor para inclinação, caso queira avaliar uma peça cujos eixos de forças serão afetados pelo ângulo.

Figura 10: Inserção de dados de carregamento
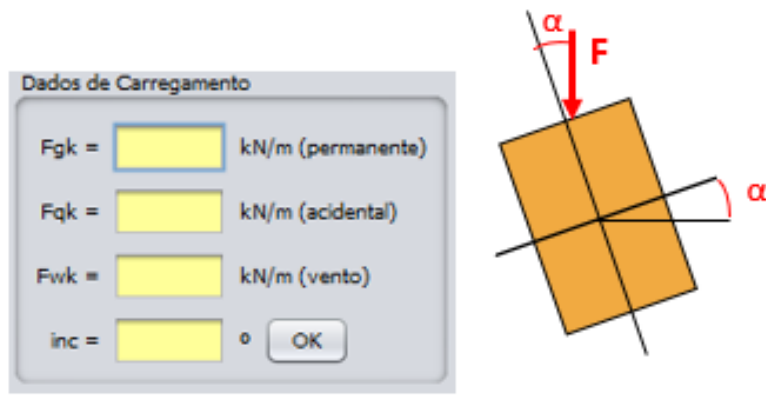

Fonte: Os autores

Se o usuário tentar inserir valores incorretos, ou ainda deixar de inserir valores, o aplicativo irá alertá-lo.

O próximo passo na sequência de cálculos é a atualização das forças de carregamento para os eixos inclinados (se foi inserido um valor para inclinação):

$$
\begin{aligned}
& g_{x}=F_{g k} * \sin \alpha, \\
& g_{y}=F_{g k} * \cos \alpha .
\end{aligned}
$$

As combinações consideradas para o cálculo da flexão na peça estão descritas a partir do item 5 da NBR 7190/2012, são: 
Comb1 - Estado Limite Último:

$$
\begin{aligned}
& q_{x d}=1.4 * g_{x}, \\
& q_{y d}=1.4 * g_{y}+0.75 * 1.4 * F_{w k} .
\end{aligned}
$$

Comb2 - Estado Limite de Utilização:

$$
\begin{aligned}
q_{x} & =g_{x}, \\
q_{y} & =g_{y}+0.2 * F_{w k} .
\end{aligned}
$$

Com os valores de forças obtidos nas combinações, é possível então calcular os resultantes de momento máximo e cortante máximo nos eixos $x$ e $y$ da peça biapoiada:

$$
\begin{aligned}
& M_{x d}=\frac{q_{x d} * L^{2}}{8} \\
& M_{y d}=\frac{q_{y d} * L^{2}}{8} \\
& V_{x d}=\frac{q_{x d} * L}{2} \\
& V_{y d}=\frac{q_{y d} * L}{2}
\end{aligned}
$$

Então, com os valores de momento máximo, podem ser definidas as tensões máximas nos respectivos eixos. Os valores de esforço cortante serão utilizados mais adiante em uma verificação

$$
\sigma_{x d}=\frac{M_{x d}}{W_{x}} ; \quad \sigma_{y d}=\frac{M_{y d}}{W_{y}} .
$$

Por fim, é necessário calcular a deformação relativa aos eixos do elemento de madeira através das equações:

$$
\begin{aligned}
& \delta_{x}=5 * \frac{L^{4}+q_{x}}{384 * E_{c 0, c f} * I_{y}}, \\
& \delta_{y}=5 * \frac{L^{4}+q_{y}}{384 * E_{c 0, c f} * I_{x}} .
\end{aligned}
$$

É finalizada a sequência de cálculos nesta aba, figura 11, trazendo ao usuário subsídios para que submeta a peça a 4 possíveis verificações, apresentadas na aba agora disponível "Verificações - Flexão".

Figura 11: Aba de Flexão

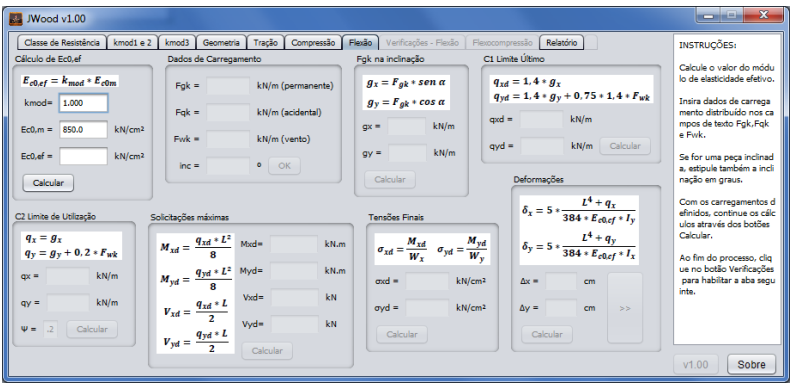

Fonte: Os autores.
As verificações apresentadas na aba seguinte podem ser feitas pelo usuário em qualquer ordem, e caso o elemento a ser calculado não passe em alguma das condições, o usuário poderá utilizar os botões "Redefinir Área"e "Redefinir Carga"para retornar às etapas anteriores na sequência de cálculo e redefinir as respectivas propriedades.

O primeiro passo na aba de Verificações-Flexão, figura 12 , é definir a resistência característica pela fórmula apresentada em (5). O coeficiente $\beta_{m}$ é calculado na sequência e é necessário para a primeira verificação: Flambagem Lateral

$$
\frac{L}{b} \leq \frac{E}{\beta_{m}} * f_{c 0 d} .
$$

- A peça é submetida à inequação a seguir e uma mensagem informa o usuário se o elemento passou na verificação

$$
\frac{L}{b} \leq \frac{E}{\beta_{m}} f_{c 0 d} .
$$

A segunda verificação é em relação à Flexão Oblíqua, através da inequação:

$$
\frac{\sigma_{x d}}{f_{c 0 d}}+0.5 * \frac{\sigma_{y d}}{f_{c 0 d}}<1 .
$$

A verificação de Tensões Cisalhantes leva em consideração os esforços máximos obtidos em (16):

$$
\frac{\frac{3}{2} * \sqrt{V_{x d}^{2}+V_{y d}^{2}}}{A} \leq 0.15 * f_{c 0 d} .
$$

Por fim, o usuário pode submeter a peça à verificação de Flexa Limite na situação biapoiada, que deve atender a seguinte inequação:

$$
\sqrt{\delta_{x^{2}}+\delta_{y^{2}}} \leq \frac{L}{300} .
$$

Com as duas abas completadas, figura 11 e 12, o aplicativo permite que o usuário determine se a peça que está dimensionando resiste ou não aos carregamentos que inseriu, podendo a qualquer momento, redefinir propriedades.

\section{Flexocompressão}

Uma opção de ferramenta adicional é disposta na aba de Flexocompressão, figura 13. Ativada quando o usuário conclui os cálculos na aba de Compressão, esta aba é dedicada especialmente a uma verificação específica no elemento biapoiado quando submetido à compressão. 
Figura 12: Aba de Verificações - Flexão

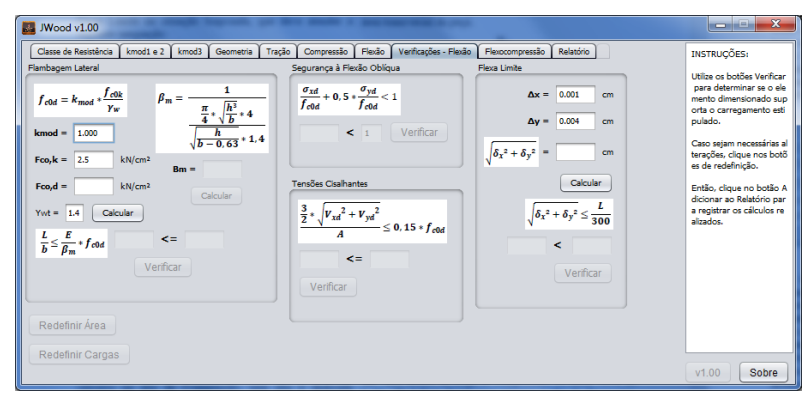

Fonte: Os autores.

Adota-se um valor de excentricidade mínima, relativo à imperfeições geométricas impostas na peça a ser verificada

$$
E_{\text {min }}=\frac{L}{300} .
$$

O usuário então deve inserir um valor referente à força de compressão, em $k_{N}$ que será aplicada axialmente e excentricamente no elemento de madeira, para que, então, possa ser obtido o esforço de momento gerado por esta interação

$$
M_{d}=N_{d} * E_{\text {min }} .
$$

Em consequência disso, o usuário poderá seguir a sequência de cálculo para determinar as tensões atuantes ocasionadas por este esforço, podendo ainda editar o campo $M_{y d}$ e estabelecer um valor próprio, se desejar,

$$
\sigma_{x d}=\frac{M_{x d}}{W_{x}} ; \quad \sigma_{y d}=\frac{M_{y d}}{W_{y}} .
$$

Em seguida, basta solicitar o cálculo da tensão relativa à força de compressão especificada pelo usuário em relação à área transversal da peça

$$
\sigma_{N c d}=\frac{N_{d}}{A}
$$

O passo seguinte consta o reconhecimento da esbeltez relativa em $x$ e em $y$, calculadas na equação (8), na aba de Compressão, figura 9, bastando o usuário clicar no botão "Verificações"para ser direcionado para uma de duas possíveis verificações, especificadas no item 5.3.6 da NBR $7190 / 2012$.

Se a esbeltez relativa de algum dos eixos for inferior ou igual a 0.3 :

$$
\begin{aligned}
& \left(\frac{\sigma_{N c, d}}{f_{c 0, d}}\right)^{2}+\frac{\sigma_{M x, d}}{f_{c 0, d}}+K_{m} \frac{\sigma_{M y, d}}{f_{c 0, d}} \leq 1, \\
& \left(\frac{\sigma_{N c, d}}{f_{c 0, d}}\right)^{2}+K_{m} \frac{\sigma_{M x, d}}{f_{c 0, d}}+\frac{\sigma_{M y, d}}{f_{c 0, d}} \leq 1 .
\end{aligned}
$$

Se ambas as esbeltezes relativas forem superiores a 0.3:

$$
\begin{gathered}
\frac{\sigma_{N c, d}}{K_{c x} f_{c 0, d}}+\frac{\sigma_{M x, d}}{f_{c 0, d}}+K_{m} \frac{\sigma_{M y, d}}{f_{c 0, d}} \leq 1 . \\
\frac{\sigma_{N c, d}}{K_{c x} f_{c 0, d}}+K_{m} \frac{\sigma_{M x, d}}{f_{c 0, d}}+\frac{\sigma_{M y, d}}{f_{c 0, d}} \leq 1 .
\end{gathered}
$$

Por fim, se o elemento não passar na verificação que foi redirecionado, o usuário pode digitar no campo em amarelo um valor menor de força de compressão, ou ainda clicar no botão "Redefinir Área"para retornar à aba de Geometria, figura 7, onde poderá modificar os valores da seção da peça.

Figura 13: Aba de Flexocompressão

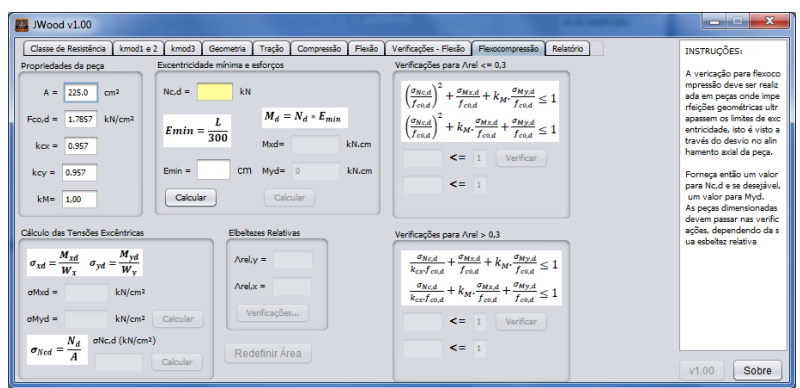

Fonte: Os autores

\section{Relatório}

Na última aba da interface da aplicação, é possível observar, a qualquer momento durante o uso do programa, através de um relatório completo de utilização, todos os passos executados pelo usuário, apresentando de maneira organizada os cálculos executados e decisões tomadas durante o uso de JWood, figura 14. Recomenda-se que os resultados utilizados e nesta aba apresentados sejam verificados por profissional habilitado para confirmação de sua veracidade.

Figura 14: Aba de Relatório

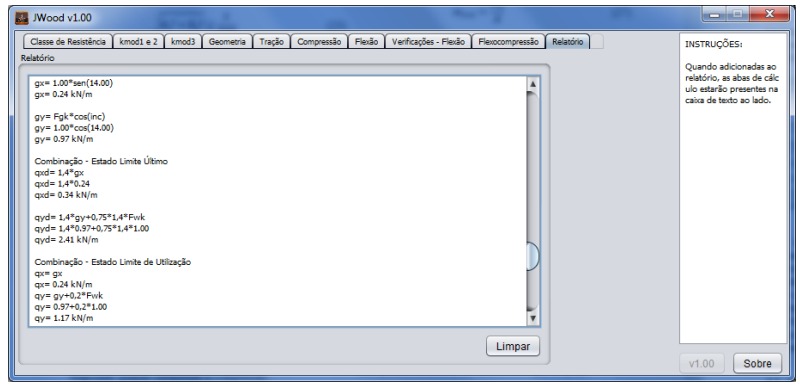

Fonte: Os autores 
Esta ferramenta possibilita que o usuário aproveite melhor seu tempo para análise e tomada de decisões, já que todas suas operações estarão disponíveis em um campo de texto ao centro da aba, transferíveis para outras ferramentas como Microsoft Word e Bloco de Notas, podendo servir também como feedback de dados inseridos e entendimento da formulação que foi aplicada durante as sequências de cálculo.

É importante constatar que o campo de texto disponibilizado na aba relatório figura 14, trata-se também de um histórico de movimentos executados, ou seja, toda vez que o usuário consolida uma operação ou toma uma decisão, isto é registrado na ordem em que foi feito. Portanto, parte também do usuário manter seus cálculos organizados.

O botão Limpar apaga todas as informações contidas no campo de texto do relatório, podendo ser clicado a qualquer momento.

\section{Conclusão}

Foi apresentado neste trabalho o programa JWood, que busca servir como ferramenta de ensino no dimensionamento e cálculo estrutural de elementos de madeira, seguindo os conceitos apresentados na NBR 7190/2012 Projetos de Estruturas de Madeira.

Os principais usos atuais de JWood pelos alunos e professores das disciplinas estruturais é no dimensionamento de estruturas como galpões e treliças de madeira. Ao ser combinado com outras ferramentas como VisualVentos e Ftool, o programa tornou-se útil tanto para o aprendizado quanto para o ensino.

Seguindo a linha de desenvolvimento de aplicativos e ferramentas computacionais ligadas ao cálculo de estruturas, JWood está disponível no site do projeto educacional Etools (www.etools.upf.br), podendo ser feito seu download gratuitamente, permitindo sua acessibilidade para todos os interessados.

É importante ressaltar que por ser um aplicativo de uso livre, nem os autores, nem a Universidade de Passo Fundo nem qualquer outra instituição relacionada ao Etools possui quaisquer deveres legais ou responsáveis relativos ao mau uso do JWood. O usuário é responsável por qualquer conclusão feita a partir da utilização do programa. Recomenda-se inclusive a leitura do manual disponível em seu download e a verificação de resultados obtidos nos cálculos do relatório através de profissionais habilitados.

Até a data de preparação deste artigo, já tinham sido baixadas mais de 500 cópias do programa.

\section{Referências}

ABNT - ASSOCIAÇÃO BRASILEIRA DE NORMAS

TÉCNICAS. NBR 7190: Projetos de estruturas de madeira. Rio de Janeiro: ABNT, 2011.

BENITTI, F. B. V.; SEÁRA, E. F. R.; SCHLINDWEIN, L. M. Processo de desenvolvimento de software educacional: proposta e experimentação. Porto Alegre: UFRGS, 2005.

CARVALHO, R. L. Programação visual com Java e IDE Netbeans. São Paulo: [s. n.], 2013.

KKRIPKA, M.; PRAVIA, Z. M. C. Proposta metodológica para o uso e desenvolvimento de ferramentas computacionais no ensino de estruturas. In: CONGRESSO BRASILEIRO DE ENSINO DE ENGENHARIA, 27., 1999, Natal. Anais... Brasília: ABENGE, 1999.

MARTHA, L. F. Ftool: interactive-graphics program for structural analysis.Versão 4.00. Rio de Janeiro: PUC, 2018. Disponível em: <http://webserver2.tecgraf.pucrio.br/ftool/>. Acesso em: 29 out. 2018.

MARTHA, L. F.; NACHT, P. K. K. Ferramenta gráficointerativa de cálculo e verificação de tensões no estado limite de serviço de vigas protendidas com pós-tração aderente para a norma brasileira através do Autodesk Robot Structural Analysys Professional. Revista IBRACON de Estruturas e Materiais, São Paulo, v. 8, n. 3, p. 427-446, jun. 2015.

MARTINS, T. F. R. M. Dimensionamento de estruturas em madeira: coberturas e pavimentos. 2010. 165 f. Dissertação (Mestrado em Engenharia Civil) - Universidade Técnica de Lisboa, Lisboa, 2010.

PRAVIA, Z. M. C.; CHIARELlO, J. A. O programa TACO: dimensionamento de elementos de estrutura de madeira segundo a NBR 7190/1997. In: CONGRESSO BRASILEIRO DE ENSINO DE ENGENHARIA, 30., 2002, Piracicaba. Anais... Piracicaba: UNIMEP, 2002.

PRAVIA, Z. M. C.; CHIARELLO, J. A. O programa VisualVentos: determinação de forças devidas ao vento em edificações de base retangular e cobertura a duas águas segundo a NBR 6123. In: CONGRESSO BRASILEIRO DE ENSINO DE ENGENHARIA, 31., 2003, Rio de Janeiro. Anais... Rio de Janeiro: IME, 2003. 
PRAVIA, Z. M. C.; PASQUETTI, E.; CHIARELLO, J.

A. VisualBarras: um software didático para o ensino de análise matricial de estruturas reticuladas planas. In: $\mathrm{CON}-$ GRESSO BRASILEIRO DE ENSINO DE ENGENHA-

RIA, 29., 2001, Porto Alegre. Anais... Porto Alegre: PUC, 2001. 